\title{
Čerenkov radiation in vacuum from a superluminal grating
}

\author{
Daigo Oue $\odot,{ }^{1}$ Kun Ding, ${ }^{1,2}$ and J. B. Pendry $\oplus^{1}$ \\ ${ }^{1}$ The Blackett Laboratory, Department of Physics, Imperial College London, Prince Consort Road, Kensington, \\ London SW7 2AZ, United Kingdom \\ ${ }^{2}$ Department of Physics, State Key Laboratory of Surface Physics, and Key Laboratory of Micro and Nano Photonic Structures \\ (Ministry of Education), Fudan University, Shanghai 200438, China
}

(Received 3 June 2021; accepted 19 January 2022; published 31 January 2022)

\begin{abstract}
Nothing can physically travel faster than light in vacuum. There are several ways proposed to bypass the light barrier and produce Čerenkov radiation ( $\check{C} R$ ) in vacuum. In this paper, we theoretically predict $\check{C}$ R in vacuum from a spatiotemporally modulated boundary. We consider the modulation of traveling wave type and apply a uniform electrostatic field on the boundary to generate electric dipoles. Since the induced dipoles stick to the interface, they travel at the modulation speed. When the grating travels faster than light, it emits ČR. In order to quantitatively examine this argument, we need to calculate the field scattered at the boundary. We utilize a dynamical differential method, which we developed in a previous paper, to quantitatively evaluate the field distribution in such a situation. We can confirm that all scattered fields are evanescent if the modulation speed is slower than light while some become propagating if the modulation is faster than light.
\end{abstract}

DOI: 10.1103/PhysRevResearch.4.013064

\section{INTRODUCTION}

Čerenkov radiation ( $\breve{\mathrm{C}})$ is radiation from a charged particle moving faster than light in a medium, which was originally observed by Čerenkov in 1934 [1] and then theoretically studied by Frank and Tamm [2]. It has been observed in various systems, including metamaterials and photonic crystals where the speed of light is effectively suppressed and hence the threshold for ČR [3-6]. There is also ČR into surface modes such as surface plasmon polaritons and Dyankov waves [7-9].

Even uncharged moving particles can emit ČR if electrically or magnetically polarized, which is closely related to the friction induced by electromagnetic fields [10-12]. Many attempts to generate $\breve{C} R$ in linear optics were focused on slowing down the speed of light by engineering the medium's dispersion relation as in the case of photonic crystals and metamaterials. On the other hand, it has been reported that ČR is emitted not only from physically moving dipoles but also from ones induced, for example, by moving light foci [13-15] and solitons in nonlinear optical fibers [16-18] or microresonators [19-21].

ČR is composed of coherent multifrequency components propagating in the same direction. It is for this property that ČR has not only attracted scientific interest but also been applied to other research fields. ČR emitted by cosmic rays has been captured in large facilities such as super-Kamiokande and played vital roles in astrophysics and high energy physics

Published by the American Physical Society under the terms of the Creative Commons Attribution 4.0 International license. Further distribution of this work must maintain attribution to the author(s) and the published article's title, journal citation, and DOI.
[22]. As mentioned above, ČR can be generated in nonlinear media. It is utilized as an optical frequency comb, as a tunable and broadband source and is expected to be utilized in spectroscopy or metrology $[19,23,24]$.

There are many reports of ČR in media as reviewed above; however, there is a limited number of studies on ČR in vacuum. This is because nothing can physically move faster than light in vacuum. One way to sidestep the light barrier in vacuum is to employ induced dipoles instead of physically moving ones. A moving light spot on a surface is one possibility to induce and mimic superluminal dipoles $[14,15]$. Another way is to introduce some background such as external electromagnetic or Chern-Simons fields. The background induces an effective refractive index to lower the ČR threshold in vacuum below the light barrier [25-28].

On the other hand, recent studies in the optics and photonics communities have shown that time-varying bulk media amplify source electromagnetic radiation [29-31] and generate light from vacuum fluctuation $[32,33]$. There are other studies revealing that temporal modulation enhances freeelectron radiation in bulk media [34-36].

In this paper, we propose a mechanism for ČR in vacuum without introducing any effective index. We consider a single interface system composed of vacuum and a dielectric (Fig. 1). By spatiotemporally modulating its interface, we can generate an interface profile of a traveling wave type,

$$
a_{\mathbf{x}}=A \sin (\mathbf{q} \cdot \mathbf{x})=A \sin \left[g\left(x-\frac{\Omega}{g} t\right)\right],
$$

where we have defined a three-component vector $\mathbf{x} \equiv$ $\{x, y, i c t\}$ and a reciprocal vector $\mathbf{q} \equiv\{g, 0, i \Omega / c\}$ for the sake of convenience, and $v_{\mathrm{ph}} \equiv \Omega / g$ is the sliding speed of the profile. Note that $\Omega$ and $g$ are independent modulation 


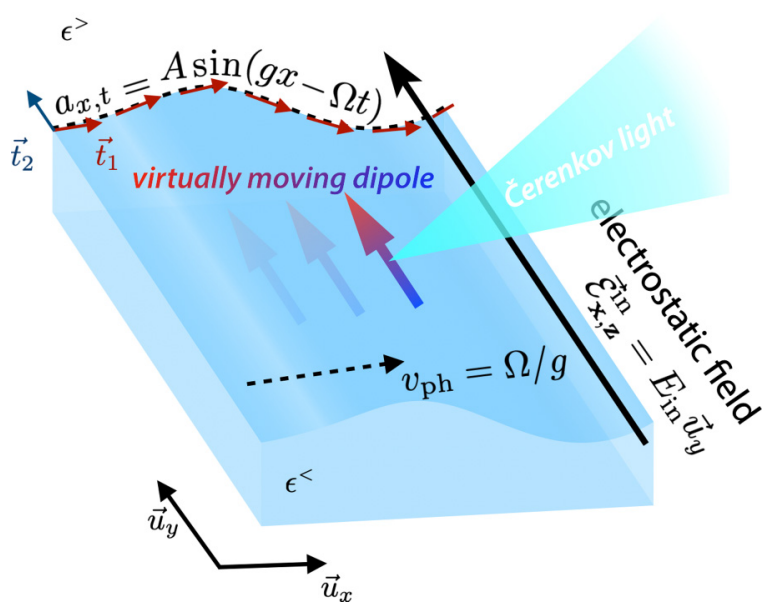

FIG. 1. Single interface system composed of a dielectric and vacuum. The interface is under the spatiotemporal modulation of traveling wave type (1). The orthonormal tangential vectors $\vec{t}_{1,2}$ can be calculated from the interface profile. The permittivities above and below the interface are denoted as $\epsilon^{>}$and $\epsilon^{<}$, respectively. We apply a uniform electrostatic field $\overrightarrow{\mathcal{E}}_{\mathbf{x}, z}^{\text {in }}$ on the modulated interface so that there are induced dipoles which travel on the interface due to its profile of the traveling wave type. The velocity of the grating $v_{\text {ph }}=\Omega / g$ can be tuned by two independent parameters so that it can exceed the speed of light. When the speed is faster than light, the induced dipoles emit Čerenkov radiation.

parameters (temporal and spatial modulation frequencies), and thus the sliding speed $v_{\mathrm{ph}}$ is not limited by the speed of light. Note also that the surface displacement speed is limited by the speed of light $(A \Omega<c)$. In other words, the upper limit of the modulation depth is determined by the temporal modulation frequency $\left(A_{\text {sup }}=c / \Omega\right)$. In the following, we are working on a regime $A \Omega \ll c$ so that we can safely neglect relativistic effects.

Since we are focusing on dielectrics, the permeability is assumed to be unity everywhere $(\mu=1)$. The permittivity is given by means of the interface profile,

$$
\epsilon_{\mathbf{x}, z}=\alpha \Theta\left(a_{\mathbf{x}}-z\right)+\epsilon^{>},
$$

where we denote the permittivities of the upper and lower media as $\epsilon^{><}$, the permittivity difference as $\alpha \equiv \epsilon^{<}-\epsilon^{>}$, and the Heaviside unit step function as $\Theta(z)$.

When an electrostatic field $\overrightarrow{\mathcal{E}}^{\text {in }}=E_{\text {in }} \vec{u}_{y}$ is applied to this configuration, electric dipoles are induced on the interface. Following the traveling type profile of the interface, the induced dipoles move virtually along the interface. If the profile velocity and hence that of the induced dipoles is faster than light, the induced dipoles may emit ČR.

Our system is periodic in the $x$ direction and in time, and thus the radiation wave number in the $x$ direction and the frequency in our system are written $k_{x, m}=k_{x}+m g$ and $\omega_{m}=\omega+m \Omega$ in accordance with the Floquet-Bloch theory, where $m=0, \pm 1, \pm 2, \ldots$ is the diffraction order. Note that here we have multifrequency radiation in contrast with ordinary diffraction. The propagation direction of the $m$ th order diffraction is $\theta_{m}^{\tau}=\cos ^{-1}\left(c k_{x, m} / \omega_{m} \sqrt{\epsilon^{\tau}}\right)$ in each medium. Since we have a uniform electrostatic field $\left(k_{x}=0, \omega=\right.$

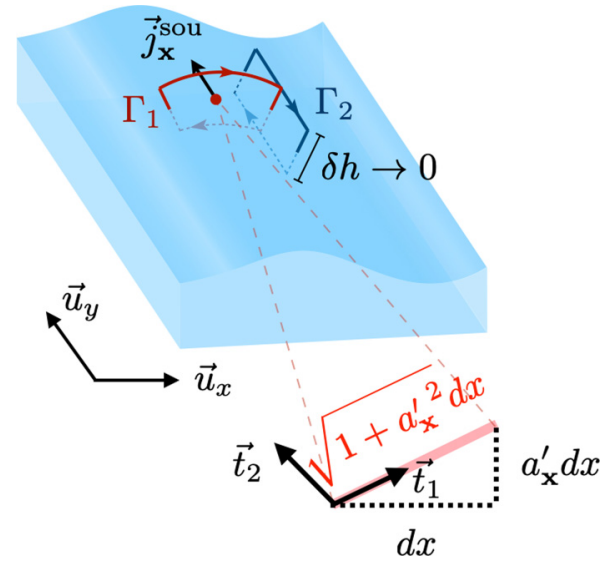

FIG. 2. Integration paths leading to the boundary conditions at the modulated interface. The modulation-induced source, $\vec{j}$ sou , comes into contribution.

0 ), all the polychromatic waves in the different diffraction orders propagate in the same direction in each medium, $\theta_{m}^{\tau}=\cos ^{-1}\left(c / v_{\mathrm{ph}} \sqrt{\epsilon^{\tau}}\right) \equiv \theta_{\check{\mathrm{CR}}}^{\tau}$. Note that, in general, permittivities are dependent on the frequency, and the propagation direction does depend on the diffraction order, i.e., $\theta_{m}^{\tau}=$ $\cos ^{-1}\left[c / v_{\mathrm{ph}} \sqrt{\epsilon^{\tau}(m \Omega)}\right]$. However, we are focusing on dielectric materials whose permittivities can be assumed to be constant numbers, and the frequency dependence can be dropped $\left[\epsilon^{\tau}(\omega) \rightarrow \epsilon^{\tau}\right]$.

\section{MODULATION-INDUCED SOURCE AT THE INTERFACE}

Here, we briefly review a dynamical differential method, which we developed in the previous work [37], and calculate the diffraction at the interface subject to modulations in space and time. Our previous work is based on the coordinate translation method originally proposed by Chandezon et al. [38,39], where they consider static corrugated interfaces and match Maxwell's boundary conditions directly at the interfaces with the help of differential geometry. Using this method, it is straightforward to take the structure of the interfaces into consideration, and it has been utilized to calculate structured surfaces of various media, including anisotropic, plasmonic, and dielectric materials [40-44]. It is also worth noting that there is a series of studies that confirm that the method works well for smooth shallow corrugations and propose possible ways to improve the method so that they can handle deep corrugation even with sharp edges [45-51]. In these works, local distortion of the coordinate systems is applied instead of the global translation of the coordinate in order to improve the convergence. Since we are interested in the time-dependent corrugation of the traveling wave type, we assume that the corrugation depth is small and safely work on the simple global coordinate translation.

In order to calculate diffraction at the structured interface, we need relevant boundary conditions. By integrating Maxwell-Heaviside equations over two kinds of path, $\Gamma_{1,2}$, which enclose the interface as shown in Fig. 2, we can derive 
the boundary conditions,

$$
\begin{aligned}
\eta \vec{t}_{1} \cdot\left(Z_{0} \overrightarrow{\mathcal{H}}_{\mathbf{x}, a_{\mathbf{x}}+0}-Z_{0} \overrightarrow{\mathcal{H}}_{\mathbf{x}, a_{\mathbf{x}}-0}\right) & =\vec{t}_{2} \cdot Z_{0} \vec{j}_{\mathbf{x}}^{\text {sou }}, \\
\vec{t}_{2} \cdot\left(\overrightarrow{\mathcal{E}}_{\mathbf{x}, a_{\mathbf{x}}+0}-\overrightarrow{\mathcal{E}}_{\mathbf{x}, a_{\mathbf{x}}-0}\right) & =0 .
\end{aligned}
$$

Here, we have a modulation-induced source term,

$$
\vec{j}_{\mathbf{x}}^{\text {sou }}=\frac{\dot{a}_{\mathbf{x}}}{c} \alpha \overrightarrow{\mathcal{E}}_{\mathbf{x}, a_{\mathbf{x}}-0},
$$

which is finite if there is time dependence (i.e., $\dot{a}_{\mathbf{x}} \neq 0$ ). This source term is responsible for radiation from the interface. Note that we have introduced the vacuum impedance $Z_{0} \equiv$ $\sqrt{\mu_{0} / \epsilon_{0}}$. The orthonormal tangential vectors of the interface, $\vec{t}_{1,2}$, can be given by means of the interface profile,

$$
\vec{t}_{1}=\frac{\vec{u}_{x}+a_{\mathbf{x}}^{\prime} \vec{u}_{z}}{\sqrt{1+a_{\mathbf{x}}^{\prime 2}}}, \quad \vec{t}_{2}=\vec{u}_{y}
$$

and the electric and magnetic fields in real space as $\overrightarrow{\mathcal{E}}_{\mathbf{x}, z}$ and $\overrightarrow{\mathcal{H}}_{\mathbf{x}, z}$. We have defined the spatial derivative of the surface profile, $a_{\mathbf{x}}^{\prime}=\partial a_{\mathbf{x}} / \partial x$. Note that we have a factor of $\eta=$ $\sqrt{1+a_{\mathbf{x}}^{\prime 2}}$ in front of $\vec{t}_{1}$ in Eq. (3), which corresponds to the spatial variation (expansion or contraction) of the differential line element due to the coordinate translation, while we do not have that factor at $\vec{t}_{2}$ because the translation does not affect the line element in the direction.

Since our system is periodic in space and time, we can substitute Floquet-Bloch type solutions in the upper and lower media, e.g., $\overrightarrow{\mathcal{E}}_{\mathbf{x}, z}=\sum_{m} E_{\mathbf{k}_{m}, z} \vec{t}_{2} e^{i \mathbf{k}_{m} \cdot \mathbf{x}} \quad\left(\mathbf{k}_{m}=\mathbf{k}+\right.$ $\left.m \mathbf{q}, \quad \mathbf{k} \equiv\left\{k_{x}, 0, i k_{0}=i \omega / c\right\}\right)$, to obtain simultaneous equations in the reciprocal space,

$$
\left(\begin{array}{cc}
\mathrm{N}_{\mathbf{k}}^{+>} & -\left(\mathrm{N}_{\mathbf{k}}^{-<}+\mathrm{L}_{\mathbf{k}}\right) \\
\mathrm{M}_{\mathbf{k}}^{+>} & -\mathrm{M}_{\mathbf{k}}^{-<}
\end{array}\right)\left(\begin{array}{l}
\mathbb{E}_{\mathbf{k}}^{-<} \\
\mathbb{E}_{\mathbf{k}}^{+>}
\end{array}\right)=\left(\begin{array}{c}
-\mathrm{N}_{\mathbf{k}}^{->} \mathbb{E}_{\mathbf{k}}^{->} \\
-\mathrm{M}_{\mathbf{k}}^{->} \mathbb{E}_{\mathbf{k}}^{->}
\end{array}\right),
$$

where we collect the modal amplitude in each diffraction order to form $\mathbb{E}_{\mathbf{k}}^{\sigma \tau}=\left(\begin{array}{lllll}\cdots & E_{\mathbf{k}_{-1}}^{\sigma \tau} & E_{\mathbf{k}_{0}}^{\sigma \tau} & E_{\mathbf{k}_{+1}}^{\sigma \tau} & \cdots\end{array}\right)^{\top}$. We have specified the upper and lower media by $\tau=\gtrless$, and the propagating direction is labeled by $\sigma= \pm$. Thus, we can regard $E_{\mathbf{k}_{m}}^{->}$as the incident components from the upper side while $E_{\mathbf{k}_{m}}^{+>}$and $E_{\mathbf{k}_{m}}^{-<}$as the diffracted components in the upper and lower sides. The effects of the source term and interface geometry are encoded in the L, M, and $\mathrm{N}$ coefficients matrices, whose elements read

$$
\begin{gathered}
{\left[\mathrm{L}_{\mathbf{k}}\right]_{l m}=\frac{\alpha}{c} \frac{(l-m) \Omega}{-K_{\mathbf{k}_{m}}^{<}} \times \operatorname{sgn}\left(\omega_{m}\right) \frac{k_{x, m}}{\left|k_{x, m}\right|} J_{l-m}\left(\phi_{\mathbf{k}_{m}}^{-<}\right),} \\
{\left[\mathrm{M}_{\mathbf{k}}^{\sigma \tau}\right]_{l m}=\frac{k_{x, m}}{\left|k_{x, m}\right|} \operatorname{sgn}\left(\omega_{m}\right) J_{l-m}\left(\phi_{\mathbf{k}_{m}}^{\sigma \tau}\right),} \\
{\left[\mathrm{N}_{\mathbf{k}}^{\sigma \tau}\right]_{l m}=\left(\frac{\sigma K_{\mathbf{k}_{m}}^{\tau}}{k_{x, m}}-\frac{(l-m) g}{\sigma K_{\mathbf{k}_{m}}^{\tau}}\right) \frac{\left|k_{x, m}\right|}{\left|k_{0, m}\right|} J_{l-m}\left(\phi_{\mathbf{k}_{m}}^{\sigma \tau}\right) .}
\end{gathered}
$$

Here, $J_{m}$ is the $m$ th order Bessel function of the first kind, and we have defined the wave number in the $z$ direction in a medium labeled by $\tau$,

$$
K_{\mathbf{k}}^{\tau} \equiv \operatorname{sgn}(\omega) \operatorname{Re} \sqrt{\epsilon^{\tau} k_{0}^{2}-k_{x}^{2}}+i \operatorname{Im} \sqrt{\epsilon^{\tau} k_{0}^{2}-k_{x}^{2}},
$$

and the corresponding propagating phase $\phi_{\mathbf{k}_{m}}^{\sigma \tau} \equiv \sigma K_{\mathbf{k}_{m}}^{\tau} A$. By truncating $\mathrm{M}, \mathrm{N}$, and $\mathrm{L}$ matrices to finite rank ones with a truncation index $m_{c}$ (i.e., $|l|,|m| \leqslant m_{c}$ ), we can numerically invert the matrix on the left-hand side and evaluate the diffraction amplitudes.

\section{III. ČERENKOV RADIATION}

We can reconstruct the diffracted field distribution in real space by substituting the diffraction amplitudes, $E_{\mathbf{k}_{m}}^{+>}$and $E_{\mathbf{k}_{m}}^{-<}$, obtained from Eq. (6) into the Floquet-Bloch series,

$$
\begin{aligned}
\overrightarrow{\mathcal{E}}_{\mathbf{x}, z>a_{\mathbf{x}}}= & \sum_{|m| \leqslant m_{c}}\left(E_{\mathbf{k}_{m}}^{->} e^{-i K_{\mathbf{k}_{m}}^{>} z}+E_{\mathbf{k}_{m}}^{+>} e^{+i K_{\mathbf{k}_{m}}^{>} z}\right) \vec{t}_{2} e^{i \mathbf{k}_{m} \cdot \mathbf{x}}, \\
& \overrightarrow{\mathcal{E}}_{\mathbf{x}, z<a_{\mathbf{x}}}=\sum_{|m| \leqslant m_{c}} E_{\mathbf{k}_{m}}^{-<} e^{-i K_{\mathbf{k}_{m}}^{<} z} \vec{t}_{2} e^{i \mathbf{k}_{m} \cdot \mathbf{x}} .
\end{aligned}
$$

Our interest is to apply a uniform electrostatic field $(\omega=$ $0, k_{x}=0$ ). In this case, we substitute $\mathbf{k}_{m}=m \mathbf{q}$. In Fig. 3, we show snapshots of the field distributions for various modulation speeds and the corresponding cross section plots in the far field region. When the modulation speed is slower than that of light in the dielectric (subluminal regime), the distribution is uniform in the far field region both on the dielectric and vacuum sides. This is because, in the subluminal regime $\left(v_{\mathrm{ph}}=\Omega / g<c / \sqrt{\epsilon}\right)$, the wave number in the $z$ direction is imaginary for each diffraction order,

$$
K_{\mathbf{k}_{m}}^{\tau}=\frac{|m| g \sqrt{\epsilon^{\tau}}}{c} i \operatorname{Im} \sqrt{v_{\mathrm{ph}^{2}}{ }^{2}-\left(\frac{c}{\sqrt{\epsilon^{\tau}}}\right)^{2}},
$$

and hence all diffracted fields exponentially decay.

On the other hand, once the modulation speed is faster than light in the dielectric and vacuum (superluminal regime), we can find the Čerenkov type patterns (i.e., propagating waves) in each medium. This is because, unlike in the subluminal regime, the wave number in the $z$ direction is real for each diffraction order. We can confirm that all diffraction modes in each medium propagate in the same direction,

$$
\tan \theta_{m}^{\tau} \equiv \frac{K_{\mathbf{k}_{m}}^{\tau}}{k_{x, m}}=\frac{\sqrt{\epsilon^{\tau}}}{c} \operatorname{Re} \sqrt{v_{\mathrm{ph}^{2}}{ }^{2}\left(\frac{c}{\sqrt{\epsilon^{\tau}}}\right)^{2}} .
$$

Note that the far right-hand side does not depend on the diffraction order $m$. Therefore, different diffraction orders with different frequencies $\omega_{m}=m \Omega$ are superposed to form pulses. This feature is more significant in the dielectric side (see the cross section plots in Fig. 3).

What we have observed here is radiation from the virtually moving dipoles induced by the electrostatic input on the interface. The evenly spaced, moving dipoles collectively emit radiation in the far field, and thus the field pattern is a plane wave. We can also mathematically understand the radiation as emission from the source term on the right-hand side of Eq. (3), which is induced by the temporal modulation of the interface $\left(\dot{a}_{\mathbf{x}} \neq 0\right)$. Since the strength of the source is proportional to the temporal modulation frequency, the emitted field is stronger as the modulation speed $v_{\mathrm{ph}}$ increases in Fig. 3.

In experiments, a series of pulses will be observed in the far field as shown in Fig. 3. Since the pulses travel in a single direction $\theta_{\check{C} \mathrm{R}}$, one can confirm that the directional (angular) dependence of the radiation peaks around $\theta=\theta_{\check{C} \mathrm{R}}$. The radiation intensity is of the order of $100 \mathrm{~mW} \mathrm{~cm}^{-2}$ when 

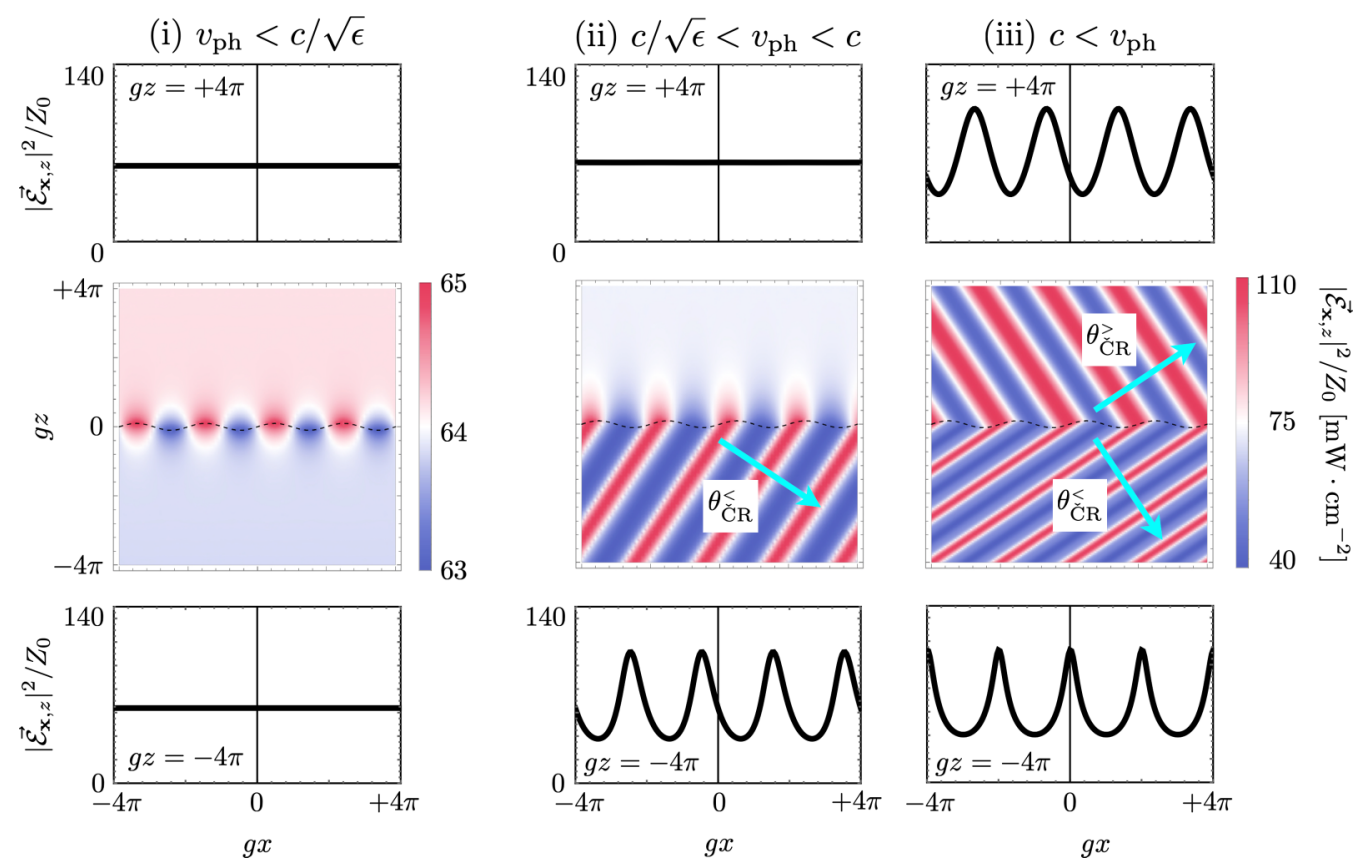

FIG. 3. Snapshots (middle row) and their cross sections (upper and lower rows) of the field distributions in the subluminal and superluminal regimes. The intensity of the total electric field $\left|\overrightarrow{\mathcal{E}}_{\mathbf{x}, z}\right|^{2}$ is plotted. (i) Subluminal regime in the dielectric and vacuum sides, $v_{\mathrm{ph}}=\Omega / g=$ $0.2 c<c / \sqrt{\epsilon}$. (ii) Superluminal in the dielectric side and subluminal in the vacuum side, $c / \sqrt{\epsilon}<v_{\mathrm{ph}}=0.8 c<c$. (iii) Superluminal regime in both sides, $c<v_{\mathrm{ph}}=1.2 c$. The modulation parameters are given as follows: the spatial frequency $g=2 \pi$ [ $\left.\mu \mathrm{m}^{-1}\right]$, the modulation depth

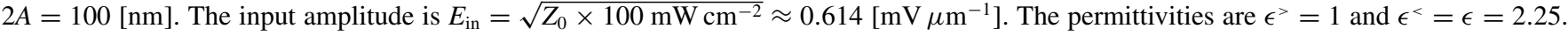
The cutoff is $m_{c}=10$ so that we take $2 m_{c}+1=21$ diffracted waves into account. The colorbars for (ii) and (iii) are common and shown on the right of (iii). Note that the scale of the colorbar of (i) is different from those of (ii) and (iii), and the horizontal axes and the vertical axes of the color plots are normalized by the spatial period $g$ of the modulation.

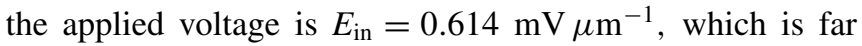
below the typical dielectric strengths of polymers and glasses $\sim 100 \mathrm{~V} \mu \mathrm{m}^{-1}$.

\section{CONCLUSIONS}

In this paper, we have proposed a mechanism for the Čerenkov radiation in a system which consists of a single interface. The spatiotemporal modulation of its interface realizes the interface profile of a traveling wave. By applying an electrostatic field, electric dipoles are induced on the interface, and move virtually on the interface due to the traveling wave profile. The profile slides at its phase velocity $v_{\mathrm{ph}}=\Omega / g$, where $\Omega$ and $g$ are independent modulation parameters, temporal and spatial modulation frequencies. Thus, the sliding speed of the profile $v_{\mathrm{ph}}$ is not limited by the speed of light. If the profile velocity and hence that of the induced dipoles is faster than light, they emit $\breve{C}$.

As for the experimental implementation, the key is the spatiotemporal modulation of the interface between two media. One can use acoustic techniques to spatiotemporally modulate the surfaces of materials. According to the studies in acoustic communities [52,53], surface displacement of the order of $10 \mathrm{~nm}$ and ultrafast modulation up to $1 \mathrm{THz}$ are achievable through acoustic-optical modulation. Using soft materials such as liquids is one way to generate large surface displacement up to micrometer scale [54-56].

Another possibility is electrostatic modulation of permittivities of atomically thin materials placed at interfaces.
Those thin materials can be regarded as infinitely thin sheets with finite conductivities [57]. Recently, several studies have revealed that the permittivity profile of graphene can be electrically modulated $[29,30,58]$. The modulation can be performed at high frequency in space and time so that graphene is another candidate to realize our proposed effects although we should take it into consideration that the optical response is dominated by two-dimensional Dirac electrons [57].

\section{ACKNOWLEDGMENTS}

D.O. is funded by the President's Ph.D. Scholarships at Imperial College London. K.D. and J.B.P. acknowledge support from the Gordon and Betty Moore Foundation. K.D. acknowledges support from Natural Science Foundation of China (Grant No. 1217040429) and Natural Science Foundation of Shanghai (Grant No. 21ZR1403700).

\section{APPENDIX A: EXTENSION TO MORE GENERAL WAVE PROFILES}

We can generalize the present analytical calculation for an arbitrary periodic profile. The key in the calculation is the expansion of the exponential function $e^{i K a_{\mathbf{x}}}$ in Eqs. (2)-(6) in the main text, which we need in order to evaluate the Fourier transform of the fields at the boundary. Let the periodicity of $a_{\mathbf{x}}$ be determined by a reciprocal vector $\mathbf{q}$. The exponential function $e^{i K a_{\mathbf{x}}}$ should also be periodic with the vector $\mathbf{q}$, and 
we can write it in terms of the Fourier series,

$$
e^{i K a_{\mathbf{x}}}=\sum_{m} A_{m}(K) e^{i m \mathbf{q} \cdot \mathbf{x}},
$$

where $A_{m}$ is the Fourier coefficient and is dependent on $K$. In the simple setup $a_{\mathbf{x}}=A \sin \mathbf{q} \cdot \mathbf{x}$ studied in the main text, the Fourier coefficients are nothing but the Bessel functions of the first kind. Thus, we can just replace the Bessel functions in the main text with general Fourier coefficients in order to deal with a more general wave type, i.e., $J_{m} \rightarrow A_{m}$.

\section{APPENDIX B: MODULATION-INDUCED SOURCE}

Since the electric flux density is the product of the permittivity and the electric field, $\overrightarrow{\mathcal{D}}=\epsilon \epsilon_{0} \overrightarrow{\mathcal{E}}$, the time variation of the permittivity produces an additional term (the source current $\left.\vec{j}=\dot{\epsilon} \epsilon_{0} \overrightarrow{\mathcal{E}}\right)$ in the Ampere-Maxwell law,

$$
\nabla \times \overrightarrow{\mathcal{H}}=\frac{\partial}{\partial t} \overrightarrow{\mathcal{D}}=\epsilon \epsilon_{0} \frac{\partial}{\partial t} \overrightarrow{\mathcal{E}}+\vec{j}
$$

The time derivative of the permittivity results in the Dirac delta function,

$$
\vec{j}=\dot{\epsilon} \epsilon_{0} \overrightarrow{\mathcal{E}}=\delta(z-a) \frac{\dot{a}}{c} \alpha \overrightarrow{\mathcal{E}}=\delta(z-a) \vec{j}^{\mathrm{sou}},
$$

that remains after integrating the Ampere-Maxwell law along a path $\Gamma_{1}$ enclosing the modulated surface as in Eq. (4) in the main text.
[1] P. A. Cherenkov, Visible light from clear liquids under the action of gamma radiation, Comp. Rend. Acad. Sci. URSS 2, 451 (1934).

[2] I. M. Frank and I. E. Tamm, Coherent visible radiation of fast electrons passing through matter, C. R. (Dokl.) Acad. Sci. URSS 14, 109 (1937)

[3] J. B. Pendry and L. M. Moreno, Energy loss by charged particles in complex media, Phys. Rev. B 50, 5062 (1994).

[4] S. Antipov, L. Spentzouris, W. Gai, M. Conde, F. Franchini, R. Konecny, W. Liu, J. Power, Z. Yusof, and C. Jing, Observation of wakefield generation in left-handed band of metamaterialloaded waveguide, J. Appl. Phys. 104, 014901 (2008).

[5] S. Xi, H. Chen, T. Jiang, L. Ran, J. Huangfu, B.-I. Wu, J. A. Kong, and M. Chen, Experimental Verification of Reversed Cherenkov Radiation in Left-Handed Metamaterial, Phys. Rev. Lett. 103, 194801 (2009).

[6] J. Tao, Q. J. Wang, J. Zhang, and Y. Luo, Reverse surfacepolariton Cherenkov radiation, Sci. Rep. 6, 1 (2016).

[7] S. Liu, P. Zhang, W. Liu, S. Gong, R. Zhong, Y. Zhang, and M. $\mathrm{Hu}$, Surface Polariton Cherenkov Light Radiation Source, Phys. Rev. Lett. 109, 153902 (2012).

[8] H. Fares and M. Almokhtar, Quantum regime for dielectric Cherenkov radiation and graphene surface plasmons, Phys. Lett. A 383, 1005 (2019).

[9] H. Hu, X. Lin, L. Jie Wong, Q. Yang, D. Liu, B. Zhang, and Y. Luo, Surface Dyakonov-Cherenkov radiation, eLight 2, 2 (2022).

[10] J. B. Pendry, Can sheared surfaces emit light?, J. Mod. Opt. 45, 2389 (1998).

[11] M. F. Maghrebi, R. Golestanian, and M. Kardar, Quantum Cherenkov radiation and noncontact friction, Phys. Rev. A 88, 042509 (2013).

[12] K. A. Milton, H. Day, Y. Li, X. Guo, and G. Kennedy, Self-force on moving electric and magnetic dipoles: Dipole radiation, Vavilov-Čerenkov radiation, friction with a conducting surface, and the Einstein-Hopf effect, Phys. Rev. Research 2, 043347 (2020).

[13] D. H. Auston, K. P. Cheung, J. A. Valdmanis, and D. A. Kleinman, Cherenkov Radiation from Femtosecond Optical Pulses in Electro-Optic Media, Phys. Rev. Lett. 53, 1555 (1984).

[14] M. Bakunov, M. Tsarev, and M. Hangyo, Cherenkov emission of terahertz surface plasmon polaritons from a superluminal optical spot on a structured metal surface, Opt. Express 17, 9323 (2009).

[15] B. Smith, J. Whitaker, and S. Rand, Steerable THz pulses from thin emitters via optical pulse-front tilt, Opt. Express 24, 20755 (2016).

[16] X. Cao and D. Meyerhofer, Soliton collisions in optical birefringent fibers, J. Opt. Soc. Am. B 11, 380 (1994).

[17] N. Akhmediev and M. Karlsson, Cherenkov radiation emitted by solitons in optical fibers, Phys. Rev. A 51, 2602 (1995).

[18] G. Chang, L.-J. Chen, and F. X. Kärtner, Highly efficient Cherenkov radiation in photonic crystal fibers for broadband visible wavelength generation, Opt. Lett. 35, 2361 (2010).

[19] V. Brasch, M. Geiselmann, T. Herr, G. Lihachev, M. H. Pfeiffer, M. L. Gorodetsky, and T. J. Kippenberg, Photonic chip-based optical frequency comb using soliton Cherenkov radiation, Science 351, 357 (2016).

[20] A. V. Cherenkov, V. E. Lobanov, and M. L. Gorodetsky, Dissipative Kerr solitons and Cherenkov radiation in optical microresonators with third-order dispersion, Phys. Rev. A 95, 033810 (2017).

[21] A. G. Vladimirov, S. V. Gurevich, and M. Tlidi, Effect of Cherenkov radiation on localized-state interaction, Phys. Rev. A 97, 013816 (2018).

[22] S. Fukuda, Y. Fukuda, T. Hayakawa, E. Ichihara, M. Ishitsuka, Y. Itow, T. Kajita, J. Kameda, K. Kaneyuki, S. Kasuga et al., The super-Kamiokande detector, Nucl. Instrum. Methods Phys. Res., Sect. A 501, 418 (2003).

[23] H. Tu and S. A. Boppart, Optical frequency up-conversion by supercontinuum-free widely-tunable fiber-optic Cherenkov radiation, Opt. Express 17, 9858 (2009).

[24] D. V. Skryabin and A. V. Gorbach, Colloquium: Looking at a soliton through the prism of optical supercontinuum, Rev. Mod. Phys. 82, 1287 (2010).

[25] C. Kaufhold and F. R. Klinkhamer, Vacuum Cherenkov radiation in spacelike Maxwell-Chern-Simons theory, Phys. Rev. D 76, 025024 (2007).

[26] A. J. Macleod, A. Noble, and D. A. Jaroszynski, Cherenkov Radiation from the Quantum Vacuum, Phys. Rev. Lett. 122, 161601 (2019).

[27] C.-Y. Lee, Cherenkov radiation in a strong magnetic field, Phys. Lett. B 810, 135794 (2020). 
[28] I. Artemenko, E. Nerush, and I. Y. Kostyukov, Quasiclassical approach to synergic synchrotron-Cherenkov radiation in polarized vacuum, New J. Phys. 22, 093072 (2020).

[29] E. Galiffi, P. A. Huidobro, and J. B. Pendry, Broadband Nonreciprocal Amplification in Luminal Metamaterials, Phys. Rev. Lett. 123, 206101 (2019).

[30] E. Galiffi, Y.-T. Wang, Z. Lim, J. B. Pendry, A. Alù, and P. A. Huidobro, Wood Anomalies and Surface-Wave Excitation with a Time Grating, Phys. Rev. Lett. 125, 127403 (2020).

[31] J. Pendry, E. Galiffi, and P. Huidobro, Gain mechanism in timedependent media, Optica 8, 636 (2021).

[32] J. Sloan, N. Rivera, J. D. Joannopoulos, and M. Soljačić, Casimir Light in Dispersive Nanophotonics, Phys. Rev. Lett. 127, 053603 (2021).

[33] J. Sloan, N. Rivera, J. D. Joannopoulos, and M. Soljačić, Controlling two-photon emission from superluminal and accelerating index perturbations, Nat. Phys. 18, 67 (2022).

[34] A. Dikopoltsev, Y. Sharabi, S. Tsesses, I. Kaminer, and M. Segev, Free-electrons radiation in a photonic time crystal, in Conference on Lasers and Electro-Optics (Optical Society of America, 2020), p. FF1Q.4.

[35] A. Dikopoltsev, Y. Sharabi, M. Lyubarov, Y. Lumer, S. Tsesses, E. Lustig, I. Kaminer, and M. Segev, Light emission by free electrons in photonic time-crystals, arXiv:2109.01203.

[36] $\mathrm{H}$. Hu, X. Lin, and Y. Luo, Free-electron radiation engineering via structured environments, Prog. Electromagn. Res. 171, 75, (2021).

[37] D. Oue, K. Ding, and J. B. Pendry, Calculating spatiotemporally modulated surfaces: A dynamical differential formalism, Phys. Rev. A 104, 013509 (2021).

[38] J. Chandezon, G. Raoult, and D. Maystre, A new theoretical method for diffraction gratings and its numerical application, J. Opt. 11, 235 (1980).

[39] J. Chandezon, M. Dupuis, G. Cornet, and D. Maystre, Multicoated gratings: A differential formalism applicable in the entire optical region, J. Opt. Soc. Am. 72, 839 (1982).

[40] J. Harris, T. Preist, and J. Sambles, Differential formalism for multilayer diffraction gratings made with uniaxial materials, J. Opt. Soc. Am. A 12, 1965 (1995).

[41] W. L. Barnes, T. W. Preist, S. C. Kitson, J. R. Sambles, N. P. K. Cotter, and D. J. Nash, Photonic gaps in the dispersion of surface plasmons on gratings, Phys. Rev. B 51, 11164 (1995).

[42] S. Kitson, W. Barnes, G. Bradberry, and J. Sambles, Surface profile dependence of surface plasmon band gaps on metallic gratings, J. Appl. Phys. 79, 7383 (1996).

[43] Y. Kitamura and S. Murakami, Hermitian two-band model for one-dimensional plasmonic crystals, Phys. Rev. B 88, 045406 (2013).
[44] G. Murtaza, A. A. Syed, and Q. A. Naqvi, Study of scattering from a periodic grating structure using Lorentz-Drude model and Chandezon method, Optik 133, 9 (2017).

[45] X. Xu and L. Li, Simple parameterized coordinate transformation method for deep- and smooth-profile gratings, Opt. Lett. 39, 6644 (2014).

[46] X. Xu and L. Li, Numerical stability of the C method and a perturbative preconditioning technique to improve convergence, J. Opt. Soc. Am. A 34, 881 (2017).

[47] X. Xu and L. Li, Numerical instability of the C method when applied to coated gratings and methods to avoid it, J. Opt. Soc. Am. A 37, 511 (2020).

[48] A. A. Shcherbakov and A. V. Tishchenko, Efficient curvilinear coordinate method for grating diffraction simulation, Opt. Express 21, 25236 (2013).

[49] A. A. Shcherbakov and A. V. Tishchenko, Generalized source method in curvilinear coordinates for 2D grating diffraction simulation, J. Quant. Spectrosc. Radiat. Transfer 187, 76 (2017).

[50] A. A. Shcherbakov, Curvilinear coordinate generalized source method for gratings with sharp edges, J. Opt. Soc. Am. A 36, 1402 (2019).

[51] S. Essig and K. Busch, Generation of adaptive coordinates and their use in the Fourier Modal Method, Opt. Express 18, 23258 (2010).

[52] C. Chenu, M.-H. Noroy, and D. Royer, Giant surface acoustic waves generated by a multiple beam laser: Application to the detection of surface breaking slots, Appl. Phys. Lett. 65, 1091 (1994).

[53] P.-A. Mante, A. Devos, and A. Le Louarn, Generation of terahertz acoustic waves in semiconductor quantum dots using femtosecond laser pulses, Phys. Rev. B 81, 113305 (2010).

[54] B. Issenmann, R. Wunenburger, S. Manneville, and J.-P. Delville, Bistability of a Compliant Cavity Induced by Acoustic Radiation Pressure, Phys. Rev. Lett. 97, 074502 (2006).

[55] B. Issenmann, A. Nicolas, R. Wunenburger, S. Manneville, and J.-P. Delville, Deformation of acoustically transparent fluid interfaces by the acoustic radiation pressure, Europhys. Lett. 83, 34002 (2008).

[56] R. W. Rambach, J. Taiber, C. Scheck, C. Meyer, J. Reboud, J. Cooper, and T. Franke, Visualization of surface acoustic waves in thin liquid films, Sci. Rep. 6, 21980 (2016).

[57] P. A. D. Gonçalves and N. M. Peres, An Introduction to Graphene Plasmonics (World Scientific, Singapore, 2016).

[58] M. M. Kashef and Z. G. Kashani, Multifunctional space-time phase modulated graphene metasurface, J. Opt. Soc. Am. B 37, 3243 (2020). 